\title{
Improper use of "radioguided occult lesion localization" (ROLL) technique leads to misleading conclusions
}

\author{
Giovanni Paganelli • Laura Gilardi • \\ Umberto Veronesi
}

Received: 4 March 2013/Accepted: 1 April 2013/Published online: 24 April 2013

(C) Springer Science+Business Media New York 2013

To the Editor,

Postma et al. [1] have compared radioguided occult lesion localization (ROLL) with wire-guided localization (WGL) in patients with non-palpable invasive breast carcinoma requiring surgery with sentinel node biopsy in a multicenter randomized controlled trial. Three hundred and fourteen patients were enrolled; 162 were allocated to ROLL and 152 to WGL. Primary outcome measures were the proportion of complete tumor excisions, the proportion of patients requiring re-excision and the volume of tissue removed. They concluded that the two procedures are comparable in terms of complete tumor excision and re-excision rates, but ROLL leads to the excision of larger tissue volumes and cannot replace WGL as the standard of care.

In our opinion, the authors committed at least three mistakes that make their work less reliable. First of all they used ${ }^{99 \mathrm{~m}} \mathrm{Tc}$-nanocolloid $\left({ }^{99 \mathrm{~m}} \mathrm{Tc}-\mathrm{NC}\right)$ as radiotracer for ROLL procedure. This is a comprehensible choice, as a single injection allowed the localization of non-palpable breast lesion and the identification of sentinel lymph node, but not the most effective one.

The ROLL technique was introduced in 1997 at the European Institute of Oncology in Milan [2]. After the first experience, which proved that this procedure allowed rapid, easy, and accurate removal of occult breast lesions [3-6], the optimal lymphoscintigraphic method to detect

G. Paganelli $(\bowtie) \cdot$ L. Gilardi

Division of Nuclear Medicine, European Institute of Oncology,

Via Ripamonti 435, 20141 Milan, Italy

e-mail: divisione.medicinanucleare@ieo.it

U. Veronesi

European Institute of Oncology, Via Ripamonti 435,

20141 Milan, Italy both occult lesions and sentinel node (that was called SNOLL: sentinel node and occult lesion localization) was evaluated [7]. Two hundred and twenty-seven patients with non-palpable breast lesions were enrolled in the study. One hundred and forty-eight patients were submitted to the ROLL procedure using macroaggregates of ${ }^{99 \mathrm{~m}} \mathrm{Tc}$-labelled human serum albumin $\left({ }^{99 \mathrm{~m}} \mathrm{Tc}-\mathrm{MAA}\right)$ injected directly into the lesion and also to lymphoscintigraphy with ${ }^{99 \mathrm{~m}} \mathrm{Tc}-\mathrm{NC}$ injected in peritumoral (62 patients) and subdermal site (86 patients). Seventy-nine patients received only an intratumoral injection of ${ }^{99 \mathrm{~m}} \mathrm{Tc}-\mathrm{NC}$ to perform both ROLL and sentinel node localization. The results showed that ROLL has a high rate of success $(94.7 \%)$, without significant differences among the groups. These findings demonstrated that NC may be used for lesion localization, but in some cases the surgeons had difficulties in delimiting the exact edge of the hot spot. This was probably due to the NC migration to the lymphatic vessels in the patients submitted to the single injection. On the contrary, MAA do not move from the injection site: consequently they do not adversely affect the radioguided tumor excision and do not determine an increase of the quantity of healthy tissue removed, as probably occurred in Postma et al. patients.

Other two factors that we believe may have affected the results are the excessive volume and amount of injected radiotracer. Indeed, the authors performed an intratumoral injection of $120 \mathrm{MBq}$ of ${ }^{99 \mathrm{~m}} \mathrm{Tc}-\mathrm{NC}$ in a volume of max $0.5 \mathrm{ml}$ of saline and this could have contributed to the excision of a larger volume of tissue. In our previous papers, we used an amount of radiotracer 12-30 times as small as Postma's one (from 3.7 to $7-10 \mathrm{MBq}$ ) in only $0.2 \mathrm{ml}$ of saline [3-7], obtaining excellent results (that were reconfirmed on more than nine hundred patients submitted to the SNOLL procedure from 1997 to 2004 [8]) and implying negligible radiation risk for patients and medical staff [9]. 
Finally, an important factor to be taken into account is that, through ROLL procedure, the exact site of the radiolabelled lesion can be checked at any time with the gamma probe during the operation. The surgeon can then determine the best incision to reach the lesion, regardless of the site of radiotracer injection. In this way a portion of breast tissue containing the lesion at its centre can be removed, guaranteeing oncological radicality and also avoiding excessive mutilation [3, 4].

After all, the ROLL procedure with direct injection of ${ }^{99 \mathrm{~m}} \mathrm{Tc}-\mathrm{MAA}$ into the lesion is easy, safe, and accurate and it can be performed in association with subdermal injection of ${ }^{99 \mathrm{~m}} \mathrm{Tc}-\mathrm{NC}$ for sentinel node detection without interference. From 1999 it represents the method of choice for accurate localization of non-palpable breast lesion in our institution, where it has been invented and performed, alone or as SNOLL, in nearly 10,000 patients (Paganelli G, Veronesi U, personal communication).

Last but not least, the technique and acronym ROLL although not registered was pioneered at IEO in 1996 and should be used as originally described [2-4]. What the socalled "ROLL study group" have done is certainly something different from the "original" ROLL technique described by our group.

Conflict of interest The authors declare that they have no conflict of interest.

\section{References}

1. Postma EL, Verkooijen HM, van Esser S et al (2012) Efficacy of 'radioguided occult lesion localisation' (ROLL) versus 'wireguided localisation' (WGL) in breast conserving surgery for nonpalpable breast cancer: a randomised controlled multicentre trial. Breast Cancer Res Treat 136:469-478

2. Paganelli G, De Cicco C, Luini A et al (1997) Radioguided surgery in non-palpable breast lesions. Eur $\mathbf{J}$ Nucl Med 24(suppl):893

3. Luini A, Zurrida S, Galimberti V et al (1998) Radioguided surgery of occult breast lesions. Eur J Cancer 34:204-205

4. Luini A, Zurrida S, Paganelli G et al (1999) Comparison of radioguided excision with wire localisation of occult breast lesions. Br J Surg 86:522-525

5. Gennari R, Galimberti V, De Cicco C et al (2000) Use of technetium-99 m-labeled colloid albumin for preoperative and intraoperative localization of nonpalpable breast lesion. J Am Coll Surg 190:692-699

6. De Cicco C, Pizzamiglio M, Trifirò G et al (2002) Radioguided occult lesion localisation (ROLL) and surgical biopsy in breast cancer. Technical aspects. Q J Nucl Med 46:145-151

7. De Cicco C, Trifirò G, Intra M et al (2004) Optimised nuclear medicine method for tumour marking and sentinel node detection in occult primary breast lesions. Eur J Nucl Med Mol Imaging 31:349-354

8. Monti S, Galimberti V, Trifirò G et al (2007) Occult breast lesion localization plus sentinel node biopsy (SNOLL): experience with 959 patients at the European institute of oncology. Ann Surg Oncol 14:2928-2931

9. Cremonesi M, Ferrari M, Sacco E et al (1999) Radiation protection in radioguided surgery of breast cancer. Nucl Med Commun 20:919-924 


\section{Reply to: Improper use of "radioguided occult lesion localization" (ROLL) technique leads to misleading conclusions}

\author{
E. L. Postma • H. M. Verkooijen • M. G. Hobbelink • \\ A. J. Witkamp - M. A. van den Bosch • \\ R. van Hillegersberg
}

To the Editor,

In a reply to our previously published paper in which we compared ROLL with WGL, Paganelli et al., question the reliability of our results. First, they state that the use of technetium nanocolloid could have caused the increase of the excised tissue volumes in the ROLL group as nanocolloid can migrate into the lymphatic vessels. Indeed, we used ${ }^{99} \mathrm{Tc}$-nanocolloid as this offers the opportunity to combine the radioguided lesion detection with the sentinel node procedure in a single injection. We consider this as the most important advantage of the ROLL procedure and therefore we included only breast cancer patients that would have to undergo a sentinel node procedure. In our trial, the gamma probe was used first to identify the sentinel node, followed by the site of the maximum count rate in the breast. The surgeon excised the breast tissue around this puntum maximum while taking the size and imaging characteristics of the tumour into consideration. The surgeon did not use a fall of radioactivity for determining the size of the excised specimen. For this reason we feel that migration of the nanocolloid could not have affected our results.

In addition, Paganelli et al. suggest that the volume and amount of injected radiotracer used in our study could have affected our results. In our study we used a radiotracer injection with a dosage of $120 \mathrm{MBq}{ }^{99 \mathrm{~m}} \mathrm{Tc}$-nanocolloid.

E. L. Postma - H. M. Verkooijen · M. G. Hobbelink

A. J. Witkamp · M. A. van den Bosch .

R. van Hillegersberg $(\bowtie)$

Department of Surgery, University Medical Centre Utrecht,

Postbus 85500, 3508 GA, Utrecht, The Netherlands

e-mail: r.vanhillegersberg@umcutrecht.nl
This is a higher dosage than used and mentioned by $\mathrm{Pa}-$ ganelli (3.7-10 MBq). In the past it has been demonstrated that a higher tracer dosage leads to an improved sentinel node identification rate [1]. For this reason we chose to use this higher amount of MBq. In the past, others have used a dosage of 123-130 MBq, demonstrating good results with regard to detection of both the tumour as the sentinel node $[2,3]$.

Regarding the volume of the injected radiotracer; we used a volume of max $0.5 \mathrm{ml}$, this is $0.1-0.3 \mathrm{ml}$ more than used in studies mentioned by Paganelli. We believe that this very small difference in volume could not have been of much influence on the excision volumes. Also, the amount of tissue excised was determined by the site of the radioactive hot spot and the mammographic tumour size.

Paganelli et al. did not mention the amount of tissue excised in their cohort of patients. It would be interesting to publish these to compare the results with our results. Maybe, their results are actually quite similar to ours. And as discussed in our paper, another explanation for the absence of superiority of ROLL over WGL found in our study could be related to the very good results obtained with WGL.

Finally, it is important to realise that our study is the first well set-up RCT comparing ROLL with WGL. Paganelli et al. did not perform a comparative study.

\section{References for Rebuttal letter}

1. Valdesolmos RA, Tanis PJ, Hoefnagel CA et al (2001) Improved sentinel node visualization in breast cancer by optimizing the colloid particle concentration and tracer dosage. Nucl Med Commun 22(5):579-586 
2. Feggi L, Basaglia E, Corcione S et al (2001) An original approach in the diagnosis of early breast cancer: use of the same radiopharmaceutical for both non-palpable lesions and sentinel node localisation. Eur J Nucl Med 28(11):1589-1596. doi:10.1007/s002590100601
3. van Rijk MC, Tanis PJ, Nieweg OE et al (2007) Sentinel node biopsy and concomitant probe-guided tumor excision of nonpalpable breast cancer. Ann Surg Oncol 14(2):627-632. doi:10.1245/ s10434-006-9070-4 